Introduction: Overweight and obesity is steadily increasing in Greek adolescents over the past years. The presence of risk factors for CVD in these children is very important for further preventive and therapeutic measures.

Aim: To investigate the prevalence of insulin resistance, hypertension dyslipidemia and hyperuricemia in overweight and obese adolescents.

Material and Method: A total of 327 adolescents (189 obese and 138 overweight - according to T Cole) (162 boys and 165 girls) aged 14 (SD 2) years (12-20) attending the obesity clinic were evaluated. Anthropometric data (height, weight), BP and laboratory investigations (fasting glucose, insulin, lipid profile, uric acid, OGTT) at presentation were included in the study.

Results: Elevated blood pressure (BP) was recorded in $32.7 \%$ of the obese and $15.3 \%$ of the overweight children $(P=0 \cdot 001) ; 25 \cdot 5 \%$ of the obese $v \cdot 12 \cdot 7 \%$ of the overweight had systolic $\mathrm{BP}>95$ th percentile $(P=0.008)$ and $20 \%$ v. $4.5 \%$ had diastolic $\mathrm{BP}>95 \%$ $(P<0.001) ; 10.9 \%$ of the obese $v .8 .4 \%$ of the overweight had elevated fasting glucose $(P=0 \cdot 474)$. The proportion of insulin resistance (HOMA-IR $>3 \cdot 16$ ) was significantly higher $(62 \cdot 2 \%)$ in obese compared with overweight children $(P=0.001) ; 7 \%$ of the obese $v .3 \%$ of the overweight had low HDL $(P=0 \cdot 12)$ and $12.6 \%$ of the obese $v .5 \cdot 2 \%$ of the overweight had triglycerides $>150 \mathrm{mg} /$ $\mathrm{dl}(P=0.009) ; 26 \cdot 3 \%$ of the obese $v .12 \%$ of the overweight had high uric acid $(P=0 \cdot 009)$.

Conclusions: The frequency of several cardiometabolic risk factors is significantly higher in obese compared with overweight Greek adolescents indicating the need for early intervention.

\title{
46 - Mild reductions in BMI Z-score through lifestyle modifica- tions seem to improve cardiovascular risk in a Portuguese pediatric population
}

\author{
H Nascimento ${ }^{1,2}$, S Rocha ${ }^{1,2}$, J Fernandes ${ }^{3}$, E Costa ${ }^{2,3}$, C Rego $^{4}$, HF Mansilha ${ }^{5}$, \\ P Rocha-Pereira ${ }^{6}$, A Quintanilha ${ }^{2,7}$, A Santos-Silva ${ }^{1,2}$ and L Belo ${ }^{1,2}$
}

'Departamento de Bioquimica, Faculdade de Farmacia, Universidade do Porto, Porto, Portugal: ${ }^{2}$ Instituto de Biologia Molecular e Celular (IBMC), Universidade do Porto, Porto, Portugal: ${ }^{3}$ Escola Superior de Biotecnologia, Universidade Catolica Portuguesa, Porto, Portugal: ${ }^{4}$ Unidade de Nutricao/Servico Pediatrico, UAG-MC, Hospital de S. Joao E.P.E., Faculdade de Medicina, Universidade do Porto, Porto, Portugal: ${ }^{5}$ Departamento Pediatrico do Hospital de Criancas D. Maria Pia, Porto, Portugal: ${ }^{6}$ Centro de Investigacao em Ciencias da Saude, Universidade da Beira Interior, Covilha, Portugal: ${ }^{7}$ Instituto de Ciencias Biomedicas Abel Salazar (ICBAS),

Universidade do Porto, Porto, Portugal

Introduction: Good lifestyle habits should be introduced early in an individual's life. We studied a natural progressive approach on changing obese children and adolescent's habits and how these changes might reflect in weight loss and cardiovascular risk markers.

Method: Obese paediatric patients from University Hospital S. Joao and of Children's Hospital Maria Pia, Porto, were asked to participate in a cross-sectional study: 148 obese children and adolescents (81 females (54.7\%); mean age: $11 \cdot 0$ years (4-16)) and thirty-three matching controls participated. Sixty obese patients also agreed to participate in a longitudinal study, with a second evaluation of cardiovascular risk markers one year after the lifestyle modification intervention. A substantial BMI reduction was defined as a decrease in BMI $Z$-score of 0.3 or more over the studied period (cut-off value).

Results: Cross-sectional study: compared with controls, obese patients presented with significantly higher homeostasis model assessment-insulin resistance (HOMA-IR), triglycerides, LDL-cholesterol, apo B, insulin and C-reactive protein concentrations, whereas their HDL-cholesterol and apo A levels were significantly lower. Longitudinal study: seventeen individuals (28.3\%) reached the BMI $Z$-score reduction cut-off value, which led to a significant reduction in triglycerides, cholesterol, LDL-cholesterol, apo B, glucose, insulin levels and HOMA-IR. The other forty-three children that did not reach the cut-off presented with a significant reduction in adiponectin values and increase in lipoprotein (a) concentration.

Conclusions: Small reductions in BMI Z-score may improve cardiovascular risk profile in obese children and adolescents, with particularly notorious effects in lipid profile and insulin sensitivity. Conversely, children bellow the cut-off presented with a worsened risk profile.

Funding: The present study was supported by a PhD grant (SFRH/ BD/61407/2009) attributed to Henrique Nascimento by Fundacao para a Ciencia e Tecnologia (FCT) - Portugal. 